José Roberto Diwan

Análise de Alternativas de Investimento na Área Petrolífera sob a Ótica das Opções Reais Embutindo a Opção do Investimento em Informação

Dissertação apresentada como requisito parcial para obtenção do grau de Mestre pelo Programa de Pósgraduação em Engenharia de Produção.

Orientador: José Paulo Teixeira 


\title{
Análise de Alternativas de Investimento na Área Petrolífera sob a Ótica das Opções Reais Embutindo a Opção do Investimento em Informação
}

\begin{abstract}
Dissertação apresentada como requisito parcial para obtenção do grau de Mestre pelo Programa de Pósgraduação em Engenharia Industrial da PUC-RIO.

Aprovada pela Comissão Examinadora abaixo assinalada:
\end{abstract}

Prof. José Paulo Teixeira Orientador

Departamento de Engenharia Industrial - PUC-RIO

Prof. Carlos Patrício Samanez

Departamento de Engenharia Industrial - PUC-RIO

Prof. Tara Baidya

Departamento de Engenharia Industrial - PUC-RIO

Prof. José Eugenio Leal

Coordenador(a) Setorial do Centro Técnico Científico - PUC-RIO

Rio de Janeiro, 18 de Março de 2004 
Todos os direitos reservados. É proibida a reprodução total ou parcial do trabalho sem autorização prévia da universidade, do autor e do orientador.

\section{José Roberto Diwan}

Graduou-se em Engenharia de Produção pela UFRJ (Universidade Federal do Rio de Janeiro) em 2001. Participou do Projeto PUC/Petrobras sobre Opções Reais, coordenado pelo engenheiro da Petrobras Marco Antônio G. Dias e pelos professores José Paulo Teixeira e Kátia Rocha durante o ano de 2003

Ficha Catalográfica

Diwan, José Roberto

Análise de alternativas de investimento na área petrolífera sob a ótica das opções reais embutindo a opção do investimento em informação / José Roberto Diwan ; orientador: José Paulo Teixeira. - Rio de Janeiro : PUC, Departamento de Engenharia Industrial, 2004.

103 f. : il. ; $30 \mathrm{~cm}$

Dissertação (mestrado) - Pontifícia Universidade Católica do Rio de Janeiro, Departamento de Engenharia Industrial.

Inclui referências bibliográficas.

1. Engenharia industrial - Teses. 2. Investimento em informação. 3. Revelação das incertezas técnicas. 4. Disponibilidade de alternativas de diferentes escalas de produção. 5. Valor do projeto. 6. Tempo de aprendizado das informações. I. Teixeira, José Paulo. II. Pontifícia Universidade Católica do Rio de Janeiro. Departamento de Engenharia Industrial. III. Título.

CDD: 658.5 


\section{Agradecimentos}

Em especial a meus pais, por tudo o que fizeram para que eu chegasse até aqui.

A minha irmã, por ter sido minha inspiração nos tempos de colégio.

A minha sobrinha, Allessandra, que vem me proporcionando momentos maravilhosos desde o início do curso, quando nasceu.

A toda minha família, inclusive àqueles que não mais estão aqui presentes.

A minha namorada, Carla, por ter sido um grande estímulo, uma ótima ajudante e uma maravilhosa companhia ao longo da realização desse trabalho.

Ao professor José Paulo Teixeira pelos conhecimentos transmitidos durante o curso e pela orientação fornecida para a realização desse trabalho.

Ao professor Tara Baidya, um professor capaz de ensinar demonstrações de fórmulas em aulas bastante divertidas.

Ao professor Carlos Patrício Samanez pelos ensinamentos que enriqueceram minha formação acadêmica.

A Marco Antônio Dias pela sugestão do tema dessa dissertação, por me disponibilizar materiais de sua autoria, bem como, pela sua atenção e capacidade.

A Edison Tito e Kátia Rocha pelas dicas e ajudas fundamentais.

Aos grandes amigos desse curso de Mestrado, em especial a Eduardo, Ernesto, Javier, Renato e Rubens pelas diversões, dúvidas e explicações compartilhadas, que tanto contribuíram para nossa formação.

À Petrobras, por tornar possível a realização desse trabalho.

À CAPES, pelo apoio financeiro.

A DEUS, pela oportunidade de nascer e ainda estar vivo.

A Mim; afinal, acho que mereço! 


\section{Resumo}

Diwan, José Roberto; Teixeira, José Paulo. Análise de alternativas de investimento na área petrolífera sob a ótica das Opções Reais embutindo a opção do investimento em informação. Rio de Janeiro, 2004. 103p. Dissertação de Mestrado - Departamento de Engenharia Industrial, Pontifícia Universidade Católica do Rio de Janeiro.

A inclusão da flexibilidade gerencial nas avaliações de projetos vem promovendo análises mais condizentes com a realidade, quando comparadas àquelas geradas pelos métodos tradicionais (VPL e TIR, por exemplo). Isso porque, de acordo com o comportamento do cenário, passa-se a considerar a possibilidade de realizações de mudanças nos planos anteriormente traçados. $\mathrm{O}$ estudo desse tema é especialmente importante em cenários que apresentam bastante incerteza associada, pesados investimentos e alto grau de irreversibilidade. A indústria petrolífera encaixa-se perfeitamente no perfil desse cenário, justificando portanto, a consideração da flexibilidade gerencial nas avaliações de seus projetos, feita através da analogia às opções financeiras. Nesse caso, devido a ordem de grandeza do investimento necessário para o desenvolvimento de um campo de petróleo, o estudo das vantagens que possam vir a ser proporcionadas por um investimento anterior em informação, visando reduzir as incertezas presentes, é um tópico que requer atenção especial, sendo então um dos objetivos dessa dissertação. Para tanto, utilizando um caso real da indústria petrolífera, foi considerada a disponibilidade de três diferentes alternativas para o desenvolvimento do campo, cada qual com sua escala. Daí, sempre assumindo cenários com incertezas técnicas e econômicas, foram feitas simulações para diferentes percentuais de revelações das incertezas (proporcionados pela informação anteriormente coletada), para diferentes custos de investimento em informação e para diferentes combinações de alternativas; simulação essa que ilustra o valor agregado pela flexibilidade criada com a adição de mais alternativas para o desenvolvimento do campo e que vem a ser outro objetivo dessa dissertação.

\section{Palavras-chave}

Investimento em informação; revelação das incertezas técnicas; disponibilidade de alternativas de diferentes escalas de produção; valor do projeto; tempo de aprendizado das informações. 


\section{Abstract}

Diwan, José Roberto; Teixeira, José Paulo (Advisor). Analysis of investment's alternatives in the oil industry through the Real Options theory considering the investment in information as an option. Rio de Janeiro, 2004. 103p. MSc. Dissertation - Departamento de Engenharia Industrial, Pontifícia Universidade Católica do Rio de Janeiro.

The Real Options Theory approaches the reality in financial analysis better than the traditional methods (NPV and TIR, for example), as it makes possible to change the plans that had already been done, according to the situation (managerial flexibility). This subject is very important, specially for projects that involve huge amount of investment, a lot of uncertainty and low reversibility. Normally, the oil industry's projects have these kind of characteristics; justifying, this way, the application of this theory. In this case, because of the huge capital needed to be invested in order to develop the oilfield, it's worthwhile to analyze the advantages that can be provided by a forward investment in information in order to reduce the technical uncertainty. This is one of the objectives of this work. Therefore, one real case of the oil industry is being used in this work, considering three different available alternatives for the development of the oilfield, which one having its own scales of production, and always adopting situations with technical and economic uncertainties. It was done simulations for different percentage of uncertainties' revelations (provided by the information obtained forwardly), for different costs of the investment in information and for different quantities of alternatives available to the manager. This last simulation shows the value added by the flexibility provided with the increasing of alternatives to develop the oilfield. This is the other objective of this work.

\section{Key-words}

Investment in information; revelation of technical uncertainties; different available alternatives in terms of production scale; projects' value; learning delay. 


\section{Sumário}

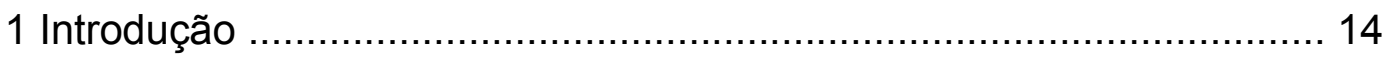

2 Teoria das Opções Reais ........................................................... 18

2.1. Teoria dos Processos Estocásticos ........................................... 20

2.2. Ferramentas de Otimização sob Incerteza .................................. 21

2.2.1. Programação Dinâmica ........................................................ 21

2.2.2. Análise por Ativos Contingenciais ........................................ 22

2.2.3. Comparação entre os Métodos ............................................ 23

3 Incertezas, Função VPL e o Investimento em Informação .................. 25

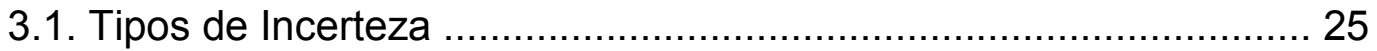

3.1.1. Incerteza de Mercado ................................................... 25

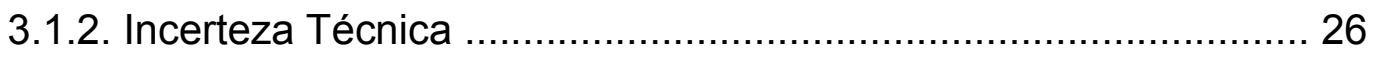

3.2. Resultado do Exercício da Opção de Desenvolvimento:a Função VPL

3.2.1. Abordagem Business Model ............................................ 28

3.3. Efeitos da Incerteza Técnica na Função VPL ................................ 30

3.3.1. Metodologia para Quantificação das Perdas .............................. 30

3.3.2. Análise do Efeito da Incerteza Técnica na Função VPL .............. 34

3.4. Investimentos em Informação e a Distribuição de Revelação ........ 34

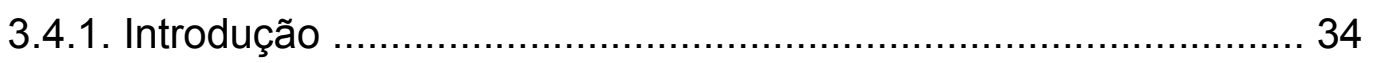

3.4.2. Investimentos em Informação ......................................... 35

3.4.3. Distribuição de Revelação ............................................... 37

3.4.3.1. As Quatro Proposições da Distribuição de Revelação ............. 38

3.4.4. O Paradoxo Gerado com o Investimento em Informação ............ 40

3.4.5. Investimentos Seqüenciais em Informação ............................ 41

4 Alternativas de Diferentes Intensidades de Investimento ................. 42

5 Apresentação e Resultados do Estudo de Caso .............................. 46 
5.1. Apresentação do Estudo de Caso .............................................. 46

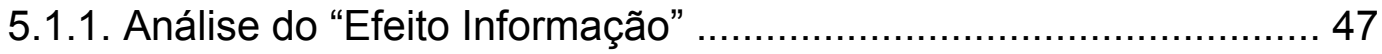

5.1.2. Análise do "Efeito Maior Disponibilidade de Alternativas" ............ 49

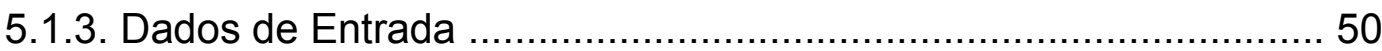

5.2. Resultados do Estudo de Caso ..................................................... 52

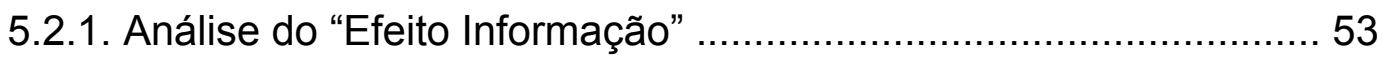

5.2.2. Análise do "Efeito Maior Disponibilidade de Alternativas" ............. 57

5.2.3. Aumento do Custo do Investimento em Informação x Redução do

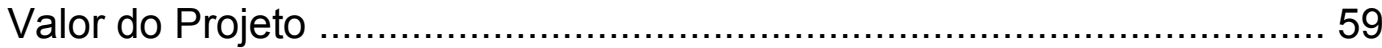

5.2.4. Valor Agregado ao Projeto e o Custo-Limite ............................... 60

5.3. Aumento do Tempo de Aprendizado x Redução do Valor do Projeto ............................. 63

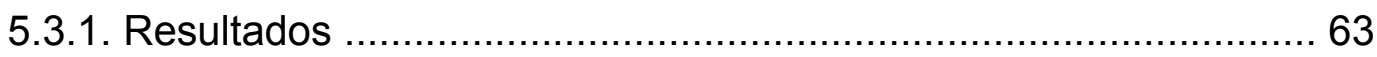

5.3.2. Reduções Numéricas e Percentuais do Valor do Projeto ............. 64

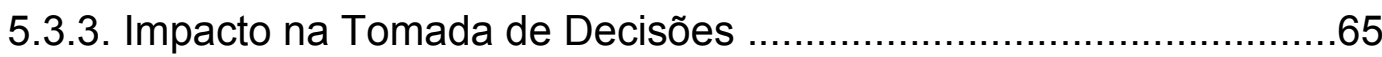

5.3.4. Custo Indiferente à Tomada de Decisão ……………………..... 67

6 Conclusões e Sugestões para Trabalhos Futuros ………………....... 69

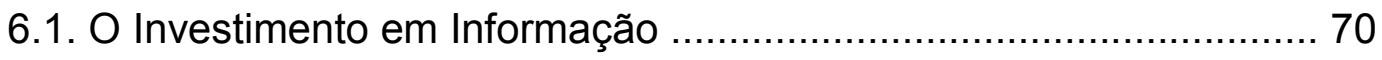

6.2. A Disponibilidade de um Leque de Alternativas .............................. 70

6.3. Sugestões para Trabalhos Futuros ……………......................... 71

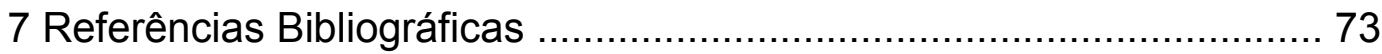

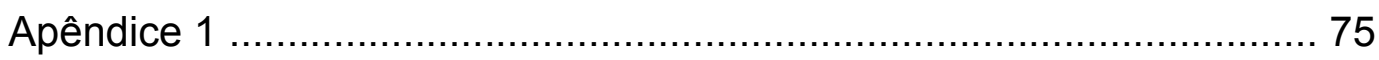

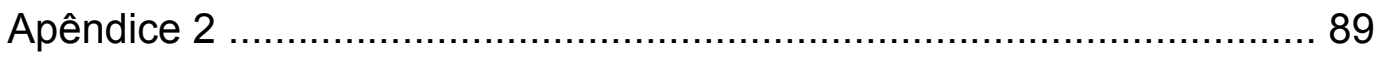




\section{Lista de tabelas}

Tabela 1 - Valor do projeto: diferentes percentuais de revelação das incertezas 48

Tabela 2 - Valor do projeto, quando não realizados investimentos em

informação 48

Tabela 3 - Valor do projeto: diferentes quantidades de alternativas disponíveis 50

Tabela 4 - Dados referentes às alternativas de produção ...................................... 51

Tabela 5 - Valor do projeto, caso Free-Rider .....................................................52

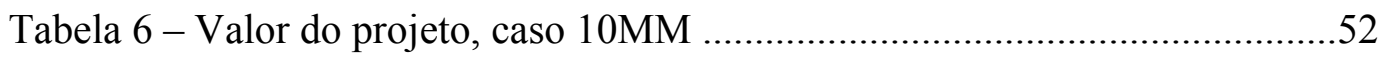

Tabela 7 - Valor do projeto, caso 30MM ……….............................................. 53

Tabela 8 - Redução do valor da opção: do caso Free-Rider para o caso 10MM 59

Tabela 9 - Redução do valor da opção: do caso 10MM para o caso 30MM ....... 59

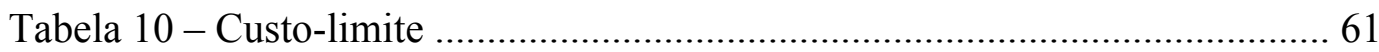

Tabela 11 - Valor do projeto, caso Free-Rider, tempo de aprendizado de 2 anos

.63

Tabela 12 - Valor do projeto, caso 10MM, tempo de aprendizado de 2 anos .... 64

Tabela 13 - Valor do projeto, caso 30MM, tempo de aprendizado de 2 anos ..... 64

Tabela 14 - Redução numérica do valor do projeto ............................................ 64

Tabela 15 - Redução percentual do valor do projeto, caso Free-Rider .............. 65

Tabela 16 - Redução percentual do valor do projeto, caso 10MM .................... 65

Tabela 17 - Redução percentual do valor do projeto, caso 30MM .................... 65

Tabela 18 - Custo-limite, tempo de aprendizado de 2 anos ...............................67

Tabela A1 - Distribuição dos valores do preço do petróleo ................................. 85

Tabela A2 - Distribuição dos valores do volume da reserva ............................... 86

Tabela A3 - Simulação dos valores do preço do petróleo .................................... 86

Tabela A4 - Simulação dos valores do volume da reserva ................................ 86

Tabela A5 - Descrição numérica da rotina do programa computacional, quando

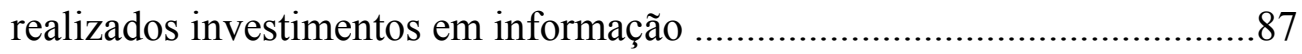

Tabela A6 - Descrição numérica da rotina do programa computacional, quando não são realizados investimentos em informação ...................................... 88

Tabela A7 - Distr. a priori do volume do campo ............................................... 91

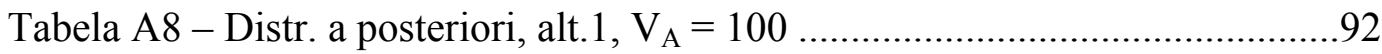


Tabela A9 - Distr. a posteriori, alt.1, $\mathrm{V}_{\mathrm{A}}=0$............................................ 93

Tabela A10 - Distr. de revelação, alt.1 ............................................................. 95

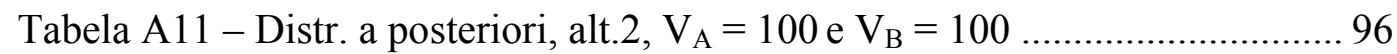

Tabela A12 - Distr. a posteriori, alt.2, $\mathrm{V}_{\mathrm{A}}=100$ e $\mathrm{V}_{\mathrm{B}}=0$............................ 96

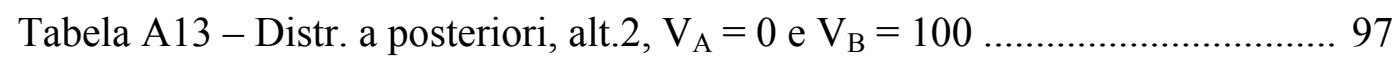

Tabela A14 - Distr. a posteriori, alt.2, $\mathrm{V}_{\mathrm{A}}=0$ e $\mathrm{V}_{\mathrm{B}}=0$.............................. 97

Tabela A15 - Distr. de revelação, alt.2 …...................................................... 98

Tabela A16 - Distr. a posteriori, alt.3, $\mathrm{V}_{\mathrm{A}}=100, \mathrm{~V}_{\mathrm{B}}=100$ e $\mathrm{V}_{\mathrm{C}}=100 \ldots \ldots \ldots . . .99$

Tabela A17 - Distr. a posteriori, alt.3, $\mathrm{V}_{\mathrm{A}}=100, \mathrm{~V}_{\mathrm{B}}=100$ e $\mathrm{V}_{\mathrm{C}}=0$.............. 99

Tabela A18 - Distr. a posteriori, alt.3, $\mathrm{V}_{\mathrm{A}}=100, \mathrm{~V}_{\mathrm{B}}=0$ e $\mathrm{V}_{\mathrm{C}}=100 \ldots \ldots \ldots \ldots . . . .99$

Tabela A19 - Distr. a posteriori, alt.3, $\mathrm{V}_{\mathrm{A}}=100, \mathrm{~V}_{\mathrm{B}}=0$ e $\mathrm{V}_{\mathrm{C}}=0$................. 100

Tabela A20 - Distr. a posteriori, alt.3, $\mathrm{V}_{\mathrm{A}}=0, \mathrm{~V}_{\mathrm{B}}=100 \mathrm{e} \mathrm{V}_{\mathrm{C}}=100 \ldots \ldots \ldots \ldots . . . .100$

Tabela A21 - Distr. a posteriori, alt.3, $\mathrm{V}_{\mathrm{A}}=0, \mathrm{~V}_{\mathrm{B}}=100 \mathrm{e} \mathrm{V}_{\mathrm{C}}=0$.................. 100

Tabela A22 - Distr. a posteriori, alt.3, $\mathrm{V}_{\mathrm{A}}=0, \mathrm{~V}_{\mathrm{B}}=0$ e $\mathrm{V}_{\mathrm{C}}=100 \ldots \ldots \ldots \ldots \ldots \ldots . . . .100$

Tabela A23 - Distr. a posteriori, alt.3, $\mathrm{V}_{\mathrm{A}}=0, \mathrm{~V}_{\mathrm{B}}=0$ e $\mathrm{V}_{\mathrm{C}}=0$..................... 101

Tabela A24 - Distr. de revelação, alt.3 ......................................................... 102 


\section{Lista de quadros}

Quadro 1 - Ordem decrescente de atratividade, caso Free-Rider ........................ 54

Quadro 2 - Aumento percentual no valor do projeto, caso Free-Rider ............... 54

Quadro 3 - Ordem decrescente de atratividade, caso 10MM ............................. 55

Quadro 4 - Aumento percentual no valor do projeto, caso 10MM .................... 55

Quadro 5 - Ordem decrescente de atratividade, caso 30MM ............................ 56

Quadro 6 - Aumento percentual no valor do projeto, caso 30MM ................... 56

Quadro 7 - Ordem decrescente de atratividade, caso Free-Rider ....................... 57

Quadro 8 - Aumento percentual no valor do projeto, caso Free-Rider ............... 57

Quadro 9 - Ordem decrescente de atratividade, caso 10MM ............................. 58

Quadro 10 - Aumento percentual no valor do projeto, caso 10MM .................. 58

Quadro 11 - Ordem decrescente de atratividade, caso 30MM .......................... 58

Quadro 12 - Aumento percentual no valor do projeto, caso 30MM .................. 58

Quadro 13 - Ordem decrescente de atratividade, caso Free-Rider, tempo de

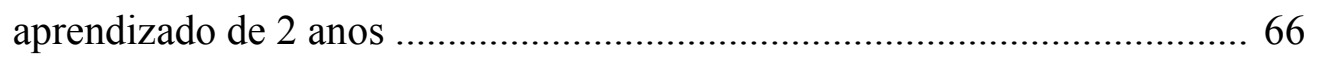

Quadro 14 - Ordem decrescente de atratividade, caso 10MM, tempo de

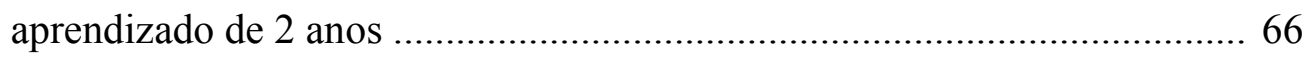

Quadro 15 - Ordem decrescente de atratividade, caso 30MM, tempo de

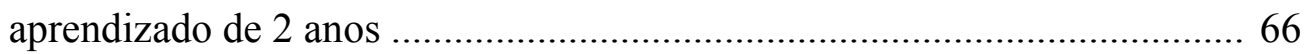




\section{Lista de figuras}

Figura 1 - Efeito da incerteza no valor da reserva desenvolvida (V) ................. 33

Figura 2 - Curvas de gatilho com regiões intermediárias de espera ................... 44

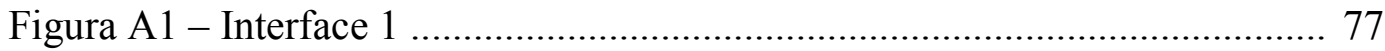

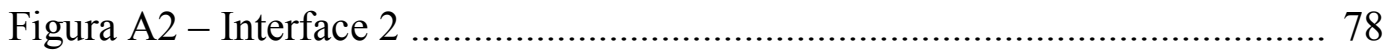

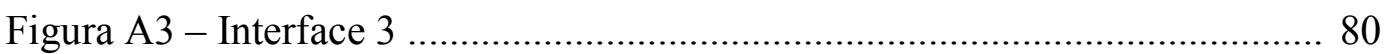

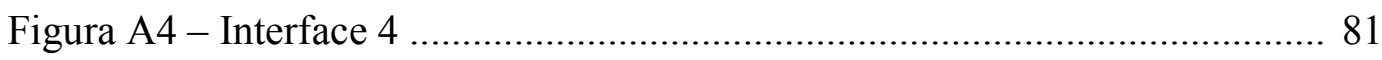

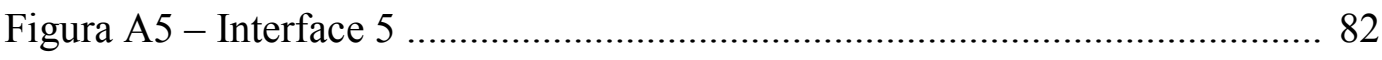

Figura A6 - Diagrama de fluxos, caso em que não há investimento em

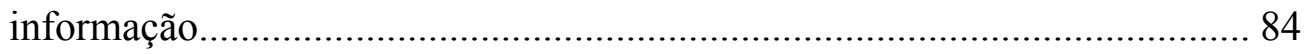

Figura A7 - Diagrama de fluxos, caso em que há investimento em informação

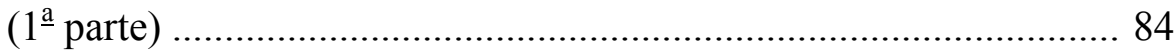

Figura A8 - Diagrama de fluxos, caso em que há investimento em informação

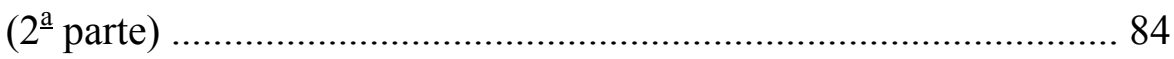

Figura A9 - Possíveis volumes das três reservas .............................................. 90

Figura A10 - Possíveis combinações dos volumes das reservas .........................91 
"Nunca ande pelo caminho traçado, pois ele conduz somente até onde os outros já foram"

Alexander Graham Bell 